\title{
The integrated assessment of occupational risks in a pharmaceutical manufacturing plant
}

\author{
Erminia Attaianese ${ }^{\mathrm{a}}{ }^{*}$ and Gabriella Duca ${ }^{\mathrm{a}}$ \\ a Deparment DICATA/LEAS, University of Naples Federico II, Via Tarsia 31, 80135, Naples, Italy
}

\begin{abstract}
Pharmaceutical plants are usually characterized by workplaces with quality levels generally higher than in other manufactoring sectors since healthcare products need to be produced in special conditions, keeping under a strong control hygiene, chemical and biological contaminants, and giving a particular emphasis on equipment maintenance. Limited physical work load, a low rate of injuries and work related pathologies together with a high level of quality process control contribute to consider pharma work conditions as generally light and safe. In this kind of contexts risk assessment cannot be based on factors individually considered, neither work load can be essentially measured on material handling and physical effort. Risk assessment and work environment improvements, on the contrary, have to be studied by an integrated view point. The paper presents methodologies and results of the applied research project IntegrARE (INTEGRated Assessment of Risks within Ergonomics) aimed at analyzing, in a big solid forms pharmaceutical plant, three different risk categories - architectural, ergonomic and active pharmaceutical ingredients risks - in order to highlight possible interactions on workers health, safety and wellbeing. After a detailed risk situations survey, singularly analyzed by a number of ad-hoc procedures, the study presents a methodology for integrated assessment of risks, aimed at identifying and evaluating integrated effects increasing or triggering risk situations for workers.
\end{abstract}

Keywords: architectural risk, ergonomic risk, active pharmaceutical ingredients, integrated risks, building

\section{Introduction}

Active dusts represent specific risk factors for workers in pharmaceutical plants, due to not only their toxicity level for health, but also, in particular environmental conditions, to their capacity to trigger fire or explosions events, also when materials used in the production process are not classified as dangerous.

Investigations following pharma accidents frequently evidence that triggering factors can be mainly related to an undervaluation of risks and, sometimes, to an uncompleted understanding of conditions affecting processes dangerousness by plant operators.

Moreover because of the potential risks, safety has been the primary concern in the pharmaceutical sector, but the rash of accidents could be largely a result of smaller, less well-regulated producers appearing in the field [1].

Consequently, for risk assessment in pharmaceutical industry, it seems to be essential to have methodologies able to analyze active dust specific risks related items, combined to other working environment components, regarding both technical both organization aspects, i.e. building features, equipment, production activities allocation as well as tasks typology and execution modalities. Risk level for workers depends mainly on the possible dynamics of incidence, that can generate acceleration or worsening of dangerous situations or, moreover, trigger further risks, that usually don't emerge by an assessment based on a singular factor analysis.

A lot of studies have been focused on occupational related musculoskeletal disorders in pharma workers [2], but less has been studied on workers health and safety assessment by an integrated point of view, considering the way in which both building and spatial components both specificities of tasks accomplished, influence the active dust related risks.

Thus the high number and typology of production buildings and spaces and the wide range of different tasks accomplished by workers in three departments of a big pharmaceutical industry, the Novartis Phar-

\footnotetext{
* Corresponding author e-mail:erminia.attaianese@unina.it
} 
ma, industrial plant of Torre Annunziata (Southern Italy), provided a unique opportunity for an applied research aimed to study a risk assessment methodology based on the integration of workplace features, ergonomic aspects and chemical factors, mainly concerning dust dynamic of diffusion.

\section{Method}

The research project IntegraARE (INTEGRated Assessment of Risks within Ergonomics) was aimed at analyzing three risk categories usually singularly assessed, but rarely fronted by an integrated point of view. In fact the evaluation of active pharmaceutical ingredients risks, that characterizes the main risk assessment in a solid forms pharmaceutical plant, was fronted considering the influence of architectonic and ergonomic aspects, in order to understand if and how, buildings features, equipment, production activities allocation, tasks typology and/or execution modalities, can be considered as risk factors for dust diffusion and concentration in the workplace [3]. Since the architectural risk survey is rarely associated with active pharmaceutical ingredients risks evaluation, a number of ad-hoc protocol were defined.

The study, performed in three years, concerned the following macro-phases:

- Plant site preliminary study and job analysis (process description and layout)

- Architectural risks survey, concerning:

* Site and building consistency study;

* Definition of architectural components/ features causing risks for workers health and safety;

* Architectural risk survey and risk localization for site main buildings.

- Ergonomic risks survey, concerning:

* Work task formalization;

* Task/Layout/Link analysis;

* Protocol for ergonomic risk assessment setting;

* Ergonomic critical point surveyed in the site;

- Active pharmaceutical ingredients risks survey;

- Integrated assessment of risks within ergonomics;

- Improvement interventions design, prioritization and implementation.

\subsection{Plant site preliminary study}

The case study is the pharma plant of NOVARTIS localized in the South of Italy. The preliminary study concerned the production process and layout description. A job analysis was done, concerning firstly workers different mansions and skills in relation to singular production phases, and then their different role within the organization, and localization in the several buildings of the plant area.

The plant area consists in a site of 200.000 square meters where only 60.000 of them are occupied by different production buildings and offices. The remaining part consists in open areas for gardens, uncovered walks and car parking.

The production is localized in two areas, including solid forms (PharmOps), and active chemicals (ChemOps). Moreover, of course, the site hosts a number of technical, accounting and management, as well as external facility functions supporting production process, substances storage places and logistic supports for contractors.

The pharmaceutical manufacturing plant has an average number of 380 employers and 120 external workers. Among them 200 workers are involved in three shift per day in the Pharm-Ops production, others are engaged in the continuous Chem-Ops process for 24 hours a day, and others are filled in support activities.

Site layout consisting of several production buildings and places, implies that the productive process phases are located in different places, strictly connected by uncovered walks so that the site can be seen as a complex workplace for a number of mansions, with indoor and outdoor areas strongly connected. For this reason a preliminary analysis of the internal-external connections flows within the site was necessary, including vehicular, pedestrian and goods pathways.

\subsection{Architectural risks survey}

Risks associated to architectonic components has been assessed considering the probability that technical and environmental components of buildings and site where production activities are carried out can generate dangerous conditions for employers health and safety, due to their features, typology and maintenance.

Particularly safety and well-being performances were studied. Safety concerns the whole of building conditions assuring an acceptable level of occupants 
protection in relation to different type of injuries, explosions, electrocutions, fire, burns and poisonings. Well-being concerns the whole of building conditions assuring users life and health. It is related to comfort and healthiness level that buildings can guarantee to their users.

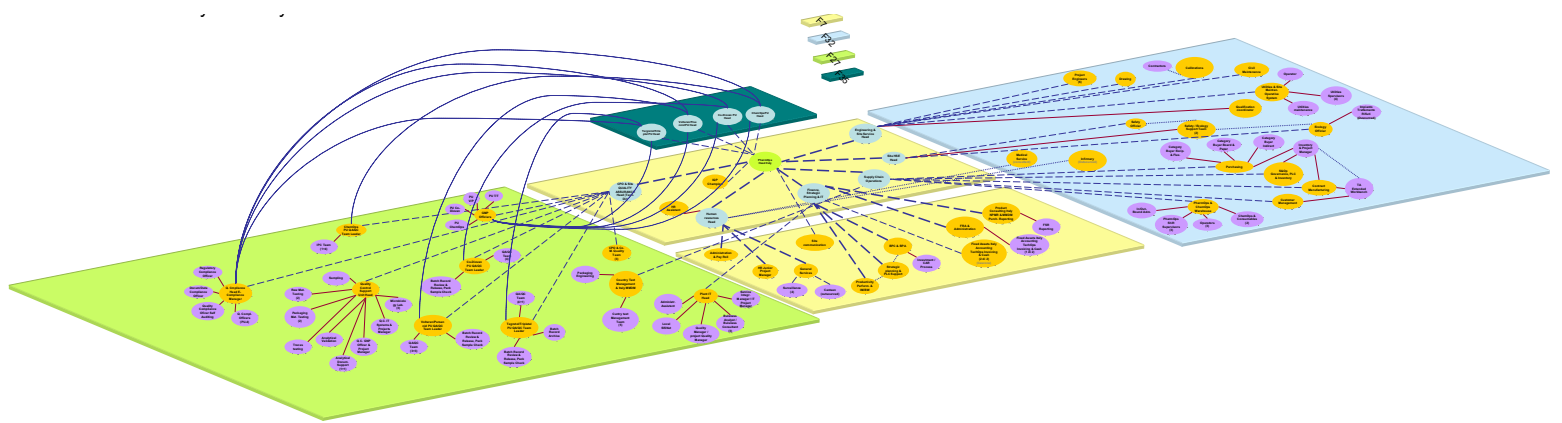

Figure 1: Layout analysis of production process

For assessing architectonic risks a number of indicators, organized in a protocol, have been identified, in order to detail the whole of building characteristics assuring that human activities are carried out in a state of safety, physical and psychological well-being. In relation to the safety, indicator areas concern on level fall, fall from height, hit, entrapment, falling object from high, vehicles impact, burn, electrocution and cutting. Moreover fire safety and building stability were considered.

In relation to health and comfort, indicators areas concern both thermo-hygrometric, visual and acoustic markers, both hygiene and indoor air quality indicators.

The formulation of the protocol for architectural risk assessment was based on the identification of building components may cause risks for occupants in relation to three elements associated to those components: their intrinsic characteristics, that can result dangerous for users in specific situations, human alterations and usage and decay, causing modifications that may completely change the component integrity and adequacy.

A protocol for architectural risk assessment has been detailed at building scale and at room scale, but also an analysis of the whole industrial area has been previously carried out. In particular a climatic analysis of the site, based on the observation of air temperature, humidity, wind speed and directions, and the evaluation of their effects on buildings performances, contributed not only to the assessment of inside workplace healthiness and comfort for employers, but mainly it helped to analyze air movements in the industrial settlement in order to understand air masses flows mixing and dilution.
Moreover environmental data gathered were also useful to energy managing of the plant.

The peculiarity of the industrial site from an environmental point of view, is the geographical location near the sea coast, thus it covers an area quite completely opened to the sea, without any obstacles to the wind. For this reason it was necessary to analyze the wind and sun radiation impact to the buildings. Moreover the presence of a urban crossing street dividing the area in two parts, created the necessity to have more pedestrian overpasses, letting the employers to reach the different areas of the industrial settlement.

This complex system of two levels pathways and the particular location induced to study first the site plan as a whole. A particular attention was reserved to the external areas, considering access and distribution flows in relation to different buildings location and functions.

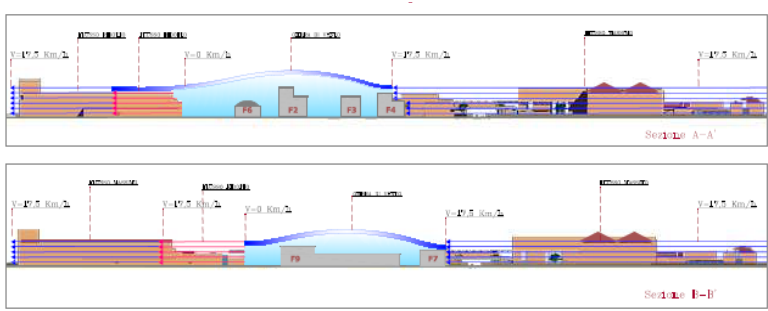

Figure 2: Particular of wind impact analysis of the plant site.

Also on the basis of those considerations the protocol for risk assessment was built considering the architectural elements as follows. At building scale were observed: plan-volumetric arrangement; orientation; façade arrangement; materials and constructive techniques; plants; green areas and natural elements 
in the surroundings; facilities in the surroundings; accesses; perceptive and image aspects; documentation check. At room scale were observed a) among construction components: walls; roofs; floorings; ceilings; stairs; HVAC; electrical system; water system; lifts; b) among room physical conditions: morphological; thermal; acoustic; lighting and chromatic; tactile and textures; electromagnetic.

\subsection{Ergonomic risks survey}

The ergonomic risks survey was based on work task formalization, by production processes breakdown. Task analysis was the key tool to understand work dynamics of employers. It was fronted in two phases: first a layout analysis for a task/functions mapping in the buildings, and then a descriptive analysis of tasks, with the aim to observe: place/places where task are carried out; operational layout; tools, equipment, bin/ sacks/ packages and substances handled; protective clothes and equipment required; movements and efforts required; moves required in the operational layout; organizational context (duration, frequency and flexibility); mental workload; link analysis; allegeable tasks flows, reflecting the production flows; observed task flows, actually performed by workers.

For assessing ergonomic risks a protocol was framed . Considered categories of ergonomic risk factors were:

- manufacture rooms features;

- manufacture pertaining spaces features;

- $\quad$ appurtenances spaces features;

- plant and machinery features;

- tools, materials and substances used and their features;

- workers movements, postures and exertions;

- activities organization and times;

- rules, procedures and individual behaviors;

- psycho-social aspects of work environment.

Each ergonomic risk marker has been associated with those operational context indicators that, determining bad human-production systems interaction models, may cause psycho-physical problems on workers. Thus the protocol check list consists in 9 sections with a total of 135 items.

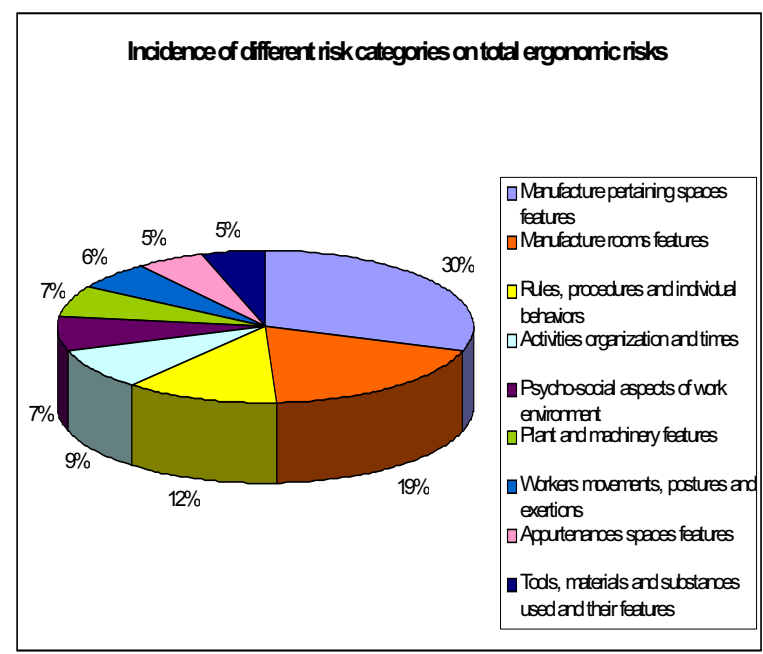

Figure 3: Incidence of different categories on total ergonomic risks.

\subsection{Active pharmaceutical ingredients risks} survey

For assessing the relation between architectonic and ergonomic aspects to active ingredients dangerousness a survey of workers exposure to dust were made, according to registration, evaluation, authorisation and restriction of chemicals (REACH) regulation and NPOHC (Novartis Pharma Occupational Health Committee).

\subsection{Integrated assessment of risks within ergonomics}

The integrated assessment of risks is aimed at analyzing how architectonic elements of workplace, ergonomic conditions and localization of active pharmaceutical ingredients are combined in the specific pharmaceutical production workplace. The assessment is finalized to understand dynamics of dust deposition/production/diffusion and consequent effects in generating increasing risks.

The integrated assessment protocol is made by a set of graphic and textual information charts, arranged in form of "thematic risk maps" of the three singular assessment applied separately. In order to synthesize and give homogeneity to the architectonic, ergonomic and chemical evaluations, each workplace of the dispensing, granulation and tabletting department has been analyzed evidencing, in the same map, the presence and the incidence on active pharmaceutical ingredients, in terms of deposition, production and diffusion. 
These risk maps, built up in relation to each specific workplace to integrated assessed contains, mainly in a graphical and visual form, the following data:

- architectonic and photographic survey of each workplace, to be able to examine architectonic elements in dimensional, morphological and finishing terms and installations (i.e. room shape and finishes, windows and doors position and dimensions, systems) identifying their specific characteristics influencing dynamics of active ingredients dust deposition/production/diffusion;

- flow chart of specific work tasks that employees perform in the workplace, in order to identify and characterize those tasks that produce (or can potentially produce) or diffuse dust of active ingredients;

- ergonomic conditions (i.e. equipment used by workers and task execution modalities) of each tasks executed by each employee in the workplace, considering their potentiality of dust production and production;

- localization of active pharmaceutical ingredients risks survey, considering environmental and personal sampling.

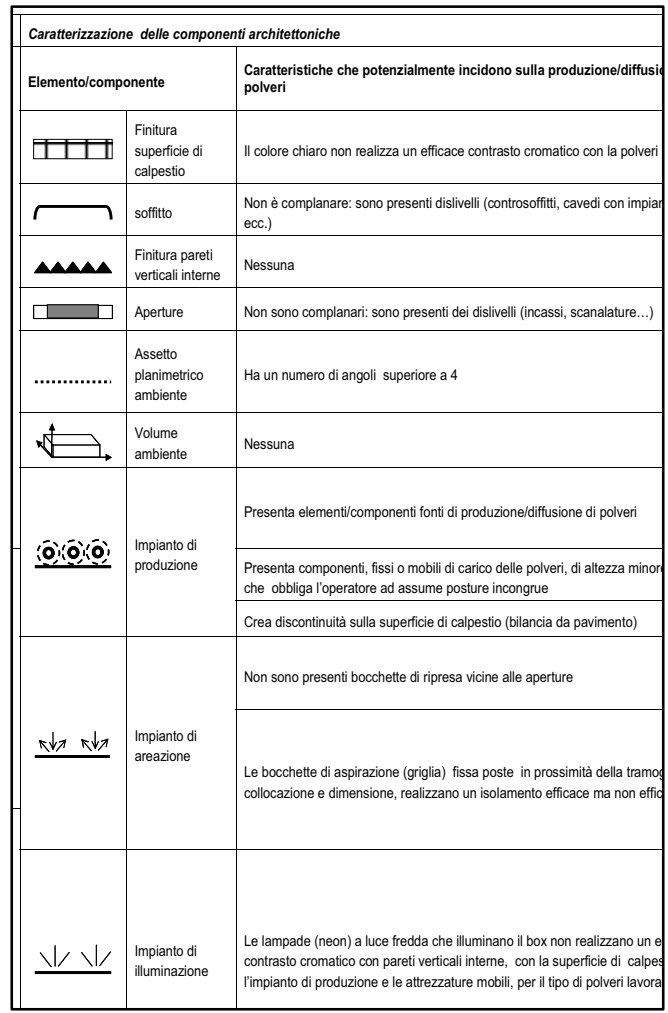

Figure 4: Sample of the key built up to represent technical data gathered on the maps. Each symbol is associated with the type of architectonic component and its potential effect on dust deposition and diffusion.

To express an effective summarization in the maps of different data previously gathered, a focus on a detailed symbolization of information has been made and organized in a key (see Fig. 4).

The integrated assessment protocol has been carried out overlapping all data layers. Since each layer reported information and data mainly expressed in a graphic form, the combined assessment has been visually highlighted observing the final map, built up by overlapping all previous singular evaluations (see the sample in Fig.5). Each area of the map evidences the presence of the number of component/element concerning characteristics of the specific workplace or performed tasks influencing active ingredients diffusion, deposit or production, giving evidence of the potentially or actually triggering risks.

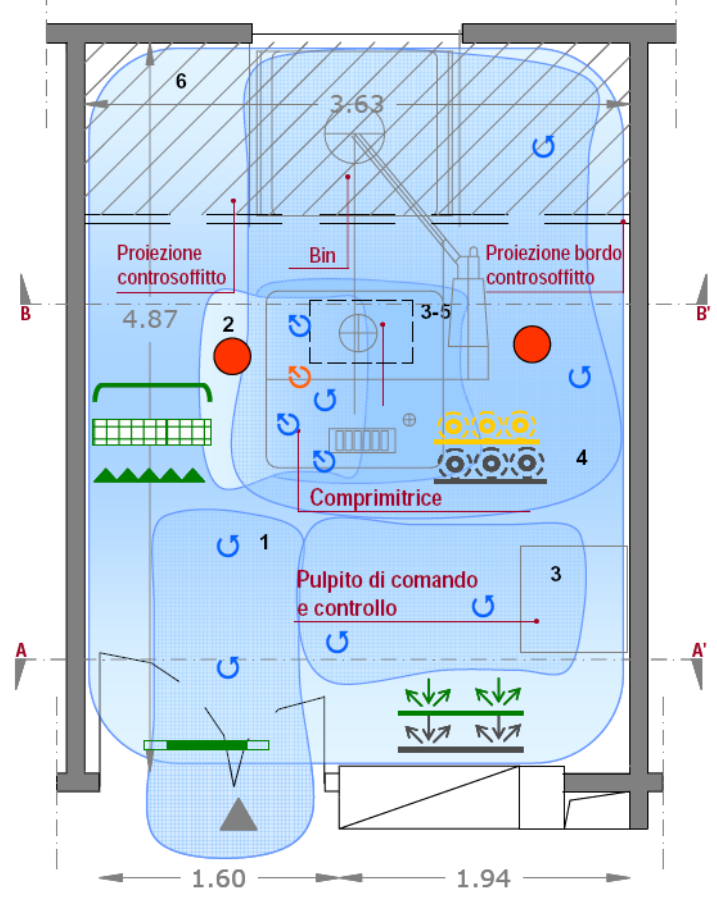

Figure 5: Sample of integrated assessment of a pharmacy box of the plant.

\section{Results}

The research produced following outputs:

- Protocol for architectural risk assessment at building scale;

- Protocol for architectural risk assessment at room scale; 
- Pharma protocol for ergonomic risk assessment (9 sections and 135 items);

- Protocol for integrated assessment of risks.

Although these protocol were designed considering the case study, they have been built up so as they can be applied to similar context to analyze.

The application of protocols were made first on the entire plant settlement, regarding architectural and ergonomic risks, emerging in consideration of environmental aspects of the site and layout. The complete set of protocols concerning single risks assessment and their successive integration were applied to three macro-phases of solid forms production process: dispensing, granulation and tabletting [3].

As result, the case study evidenced several outcomes, concerning relationship between task actual accomplishment, workspaces layout, equipment and workers behavior.

One of the main results evidences that in the pharmaceutical plant the incidence of occupational musculoskeletal disorders is not relevant for workers, although uncomfortable working position in workplaces for monitoring tasks in granulation and tabletting give good reasons to workers to stay in corridors, obstructing man/vehicles flows, decreasing monitoring tasks effectiveness and increasing psycho-physical discomfort.

On the other hand more critical points derive from integration of aspects related to features of physical environment and organization, first of all about layout. In fact workers allocation does not fit processes workflow. Workspaces layouts and dimensions give constraints to manufacturing activities, causing inappropriate behaviors and psychosocial risks for workers. The combination of corridor dimensions, path organization and mixed uses of corridors (AGV, manned vehicles and pedestrians) together with worker habits causes a high risk of impact/trip with vehicles. Functional and dimensional inadequacy of pass-boxes is a reason for safety rules (protective clothes) infringement. Functional and dimensional inadequacy of pass-boxes in dispencing is a reason for safety rules (protective clothes) infringement. Inadequacy of some of provided tools (i.e. drum-lifter, forklift in dispencing and spatula in granulation) requires unnecessary attention, time and/or physical efforts. Trolleys lifting/pulling can be very critical (due to weight, distance, stability and handling) in some specific cases (tabletting)

Materials, colors and textures of architectural finishes are inadequate to tasks, with consequences on task effectiveness and visual and psychological well- being of workers; waiting times, for example, increase dissatisfaction in workers and decrease their team spirit.

Moreover the study highlighted an increasing levels for all existing risks (workers distraction, path obstruction, environment diseases, etc.) due to freedom in length and rhythm of breaks, together with the habit to stay in walkway areas.

\section{Discussion}

The study demonstrates that in pharmaceutical plant an integrated assessment of risks may give evidence to a lot of critical situations not simply highlighted by a traditional approach. This is because architectonic features mainly influence ergonomic performances of workers executed activities, both in terms of plant and building functions location, to assure effective links in a complex context like the pharma one, both in terms of materials, colors and textures of architectural finishes, that may result inadequate to tasks, with consequences on task effectiveness and visual and psychological well-being of workers.

Moreover an integrated approach, combining how activities are executed with space and volumes arrangements and finishing show up, especially at room scale, how dynamics of dust diffusion, deposit or production may be influenced by the context, not only in pharmaceuticals, but also in food, chemical and cosmetics industries.

\section{References}

[1] A-S. Nyssen and V. Cote,. Motivational mechanisms at the origin of control task violations: An analytical case study in the pharmaceutical industry. Ergonomics, Vol. 53, Issue 9 (2010), . 1076-1084

[2] A. S. Yagjian, Risk assessment and application of engineering controls to reduce the number of occupational related musculoskeletal disorders in a large pharmaceutical production facility. Human Factors and Ergonomics Society Annual Meeting Proceedings, Industrial Ergonomics (2010), 1195-1199

[3] E. Attaianese, L. Attaianese, N., Coppola, R., d'Angelo, G. De Margheriti, G. Duca and E. Russo, The integration of environment, health and safety in evaluating ergonomic risks in solid forms pharmaceutic company (poster). Book of abstracts, 8th International Scientific Conference IOHA- International Occupational Hygiene Association 2010, (2010) 181-182

\footnotetext{
Editorial note for Italian readership: paragraphs from 1 to 2.3 can be attributed to E.A, whilst paragraphs from 2.4 to 4 can be attributed to G.D.
} 